Proyecciones Journal of Mathematics

Vol. 33, No 3, pp. 315-324, September 2014.

Universidad Católica del Norte

Antofagasta - Chile

\title{
Generalized $b$-closed sets in ideal bitopological spaces
}

\author{
Binod Chandra Tripathy \\ Institute of Advanced Study in Sc. and Tech., India \\ and \\ Diganta Jyoti Sarma \\ Central Institute of Technology, India \\ Received : April 2014. Accepted : May 2014
}

\begin{abstract}
In this article we introduce the concept of generalized b-closed sets with respect to an ideal in bitopological spaces, which is the extension of the concepts of generalized b-closed sets.
\end{abstract}

Key words and phrases: Bitopological spaces; $(i, j)-I g b$-closed sets; $(i, j)$-Igb-open sets; $(i, j)$ - gb-closed sets; $(i, j)$ - gb-open sets.

AMS Classification No: 54A10; 54C08; 54C10; $54 D 15$. 


\section{Introduction.}

The concept of bitopological spaces $\left(X, \tau_{1}, \tau_{2}\right)$ was introduced by Kelly [6]. The bitopological spaces are equipped with two arbitrary topologies $\tau_{1}$ and $\tau_{2}$. The concept of ideals has been applied in topological spaces and studied by Kuratowski [7], Vaidyanathasamy [17] and Jankovic and Hamlett [5] and others.

An ideal $I$ on a non-empty set $X$ is a collection of subsets of $X$ which satisfies $(i) A \in I$ and $B \subset A$ implies $B \in I$ and $(i i) A \in I$ and $B \in I$ implies $A \cup B \in I$. The notion of ideal has been applied for investigations in different directions. In sequence spaces ideal convergence has recently been studied by Tripathy and Hazarika [9], Tripathy and Mahanta [10], Tripathy etal. [16] and many others.

If $I$ is an ideal on $X$, then $\left(X, \tau_{1}, \tau_{2}, I\right)$ is called an ideal bitopological space. Andrijevic [3] introduced the notion of $b$-open sets in topological spaces. Later on this notion has been extended to bitopological setting by Abo Khadra and Nasef [1], Al-Hawary and Al-Omari [2] and many others. Recently, Sarsak and Rajesh [8], Tripathy and Sarma ([12], [13], [14]) have done some works on bitopological spaces using this notion. During recent years many topologists were interested in the study of different types of generalized closed sets. Mean while Fukutake [4] introduced the concept of generalized closed sets in bitopological spaces. On the other hand Tripathy and Sarma [15] introduced the notion of generalized $b$-closed sets in bitopological spaces and studied their basic properties. Recently different properties of the mixed topological spaces have been investigated from fuzzy settings by Tripathy and Ray [11] and others.

In this paper we introduce generalized $b$-closed sets with respect to an ideal in bitopological spaces and have studied some of its basic properties.

\section{Preliminaries.}

Throughout the paper $\left(X, \tau_{1}, \tau_{2}\right)$ denotes a bitopological space on which no separation axioms are assumed and $\left(X, \tau_{1}, \tau_{2}, I\right)$ be an ideal bitopological space, where $i, j \in\{1,2\}, i \neq j$. Let $A$ be a subset of $X$. 
We use the following notations.

(i) $A$ is open with respect to $\tau_{i}$ if and only if $A$ is $i$-open in $\left(X, \tau_{1}, \tau_{2}, I\right)$.

(ii) $A$ is closed with respect to $\tau_{i}$ if and only if $A$ is $i$-closed in $\left(X, \tau_{1}, \tau_{2}, I\right)$.

Now we list some known definitions and results those will be used throughout this article.

The following definitions and results are due to Al-Hawary and AlOmari [2].

Definition 2.1. A subset A of a bitopological space $\left(X, \tau_{1}, \tau_{2}\right)$ is said to be

(i) $(i, j)-b$-open if $A \subset \tau_{i}-\operatorname{int}\left(\tau_{j}-\operatorname{cl}(A)\right) \cup \tau_{j}-\operatorname{cl}\left(\tau_{i}-\operatorname{int}(A)\right)$.

(ii) $(i, j)-b$-closed if $\tau_{i}-\operatorname{cl}\left(\tau_{j}-\operatorname{int}(A)\right) \cap \tau_{j}-\operatorname{int}\left(\tau_{i}-\operatorname{cl}(A)\right) \subset A$. By $(i, j)$ we mean the pair of topologies $\left(\tau_{i}, \tau_{j}\right)$.

Definition 2.2. Let $A$ be a subset of a bitopological space $\left(X, \tau_{1}, \tau_{2}\right)$.

(i) The $(i, j)-b$-closure of $A$ denoted by $(i, j)-b c l(A)$, is defined by the intersection of all $(i, j)-b$-closed sets containing $A$.

(ii) The $(i, j)-b$-interior of $A$ denoted by $(i, j)-b i n t(A)$, is defined by the union of all $(i, j)-b$-open sets contained in $A$.

Lemma 2.1. Let $\left(X, \tau_{1}, \tau_{2}\right)$ be a bitopological space and $A$ be a subset of $X$. Then

(i) $(i, j)-\operatorname{bint}(A)$ is $(i, j)-b$-open.

(ii) $(i, j)-b c l(A)$ is $(i, j)-b$-closed.

(iii) $A$ is $(i, j)-b$-open if and only if $A=(i, j)-\operatorname{bint}(A)$.

(iv) $A$ is $(i, j)-b$-closed if and only if $A=(i, j)-b c l(A)$.

Lemma 2.2. Let $\left(X, \tau_{1}, \tau_{2}\right)$ be a bitopological space and $A$ be a subset of 
$X$. Then

(i) $x \in(i, j)-b c l(A)$ if and only if for every $(i, j)-b$-open set $U$ containing $x, U \cap A \neq \emptyset$.

(ii) $x \in(i, j)-\operatorname{bint}(A)$ if and only if there exists an $(i, j)-b$-open set $U$ such that $x \in U \subset A$.

(iii) If $A \subset B$, then $(i, j)-\operatorname{bint}(A) \subset(i, j)-\operatorname{bint}(B)$ and $(i, j)-b c l(A) \subset$ $(i, j)-b c l(B)$.

The following result is due to Sarsak and Rajesh [8].

Lemma 2.3. Let $\left(X, \tau_{1}, \tau_{2}\right)$ be a bitopological space and $A$ be a subset of $X$. Then

(i) $X-(i, j)-\operatorname{bint}(A)=(i, j)-b c l(X-A)$.

(ii) $X-(i, j)-b c l(A)=(i, j)-\operatorname{bint}(X-A)$.

The following definition is due to Tripathy and Sarma [15].

Definition 2.3. A subset A of a bitopological space $\left(X, \tau_{1}, \tau_{2}\right)$ is said to be $(i, j)$-generalized $b$-closed (in short, $(i, j)$-gb-closed) set if $(j, i)-b c l(A) \subset U$ whenever $A \subset U$ and $U$ is $\tau_{i}$-open in $X$.

\section{3. $(i, j)-I$-generalized $b$-closed Sets}

Definition 3.1. Let $\left(X, \tau_{1}, \tau_{2}, I\right)$ be an ideal bitopological space. A subset $A$ of $X$ is said to be $(i, j)-I$-generalized $b$-closed (in short, $(i, j)-I g b$ closed) set if $(j, i)-b c l(A) \backslash B \in I$ whenever $A \subset B$ and $B$ is $\tau_{i}$-open in $X$, for $i, j=1,2$ and $i \neq j$.

Theorem 3.1. Every $(i, j)-g b$-closed set is $(i, j)-I g b$-closed.

Proof. Easy, so omitted.

Remark 3.1. The converse of the above Theorem is not necessarily true. 
This is clear from the following example.

Example 3.1. Let $X=\{a, b, c\}$, consider the topologies $\tau_{1}=\{\emptyset,\{a\}, X\}$, $\tau_{2}=\{\emptyset,\{a\},\{a, b\}, X\}$ and $I=\{\emptyset,\{b\},\{c\},\{b, c\}\}$. Here $\{a\}$ is $(1,2)-I g b$ closed set but not $(1,2)$-gb-closed since $(2,1)-b c l(\{a\})=X$ not a subset of $\{a\}$.

Theorem 3.2. Let $\left(X, \tau_{1}, \tau_{2}, I\right)$ be an ideal bitopological space. If $A$ is $(i, j)-I g b$-closed and $A \subset B \subset(j, i)-b c l(A)$ in $X$, then $B$ is $(i, j)-I g b$ closed in $X$, where $i, j=1,2$ and $i \neq j$.

Proof. Let $B \subset V$ and $V$ is $\tau_{i}$-open. Since $A \subset B \subset(j, i)-b c l(A)$, we have $A \subset V$. By hypothesis $(j, i)-b c l(A) \backslash V \in I$. Further $B \subset$ $(j, i)-b c l(A)$ implies that $(j, i)-b c l(B) \backslash V \subset(j, i)-b c l(A) \backslash V \in I$. Thus $(j, i)-b c l(B) \backslash V \in I$. Consequently $B$ is $(i, j)-I g b$-closed.

Theorem 3.3. Union of two $(i, j)-I g b$-closed sets in an ideal bitopological space $\left(X, \tau_{1}, \tau_{2}, I\right)$ is also $(i, j)-I g b$-closed.

Proof. Let $A$ and $B$ be two $(i, j)-I g b$-closed sets with $A \cup B \subset V$, where $V$ is $\tau_{i}$-open. Clearly $A \subset V$ and $B \subset V$. Since $A$ and $B$ are $(i, j)-I g b$-closed, we have $(j, i)-b c l(A) \backslash V \in I$ and $(j, i)-b c l(B) \backslash V \in I$. Now $(j, i)-b c l(A \cup B) \backslash V=((j, i)-b c l(A) \cup(j, i)-b c l(B)) \backslash V=$ $((j, i)-b c l(A) \backslash V) \cup((j, i)-b c l(B) \backslash V) \in I$. Thus $(j, i)-b c l(A \cup B) \backslash V \in I$ and hence $A \cup B$ is $(i, j)-I g b$-closed set.

Remark 3.2. The intersection of two $(i, j)$ - $I g b$-closed sets is not necessarily a $(i, j)-I g b$-closed set is clear from the following example.

Example 3.2. Let $X=\{a, b, c\}$, consider the topologies $\tau_{1}=\{\emptyset,\{a\}, X\}$, $\tau_{2}=\{\emptyset,\{a\},\{a, b\}, X\}$ and $I=\{\emptyset\}$. Here $\{a, b\}$ and $\{a, c\}$ are $(1,2)-I g b$ closed sets but $\{a, b\} \cap\{a, c\}=\{a\}$ is not $(1,2)$-Igb-closed.

Theorem 3.4. Let $\left(X, \tau_{1}, \tau_{2}, I\right)$ be an ideal bitopological space. Suppose $A$ is $(i, j)-I g b$-closed in $X$ and $A \subset Y \subset X$. Then $A$ is $(i, j)-I g b$ closed relative to the subspace $Y$ of $X$ and with respect to the ideal $I_{Y}=$ $\{P \subset Y: P \in I\}$.

Proof. Let $V$ be $\tau_{i}$-open in $X$ and $A \subset Y \cap V$. Therefore we have $A \subset V$. 
Since $A$ is $(i, j)-I g b$-closed, therefore we have $(j, i)-b c l(A) \backslash V \in I$. Further we see that $((j, i)-b c l(A) \cap Y) \backslash(Y \cap V)=((j, i)-b c l(A) \backslash V) \cap Y \in I_{Y}$. Thus for $A \subset Y \cap V$ and $V$ is $\tau_{i}$-open, we have $((j, i)-b c l(A) \cap Y) \backslash(Y \cap V) \in I_{Y}$. Hence $A$ is $(i, j)-I g b$-closed relative to the subspace $\left(Y, \tau_{1}\left|Y, \tau_{2}\right| Y\right)$.

Definition 3.2. Let $\left(X, \tau_{1}, \tau_{2}, I\right)$ be an ideal bitopological space. A subset $A$ of $X$ is said to be $(i, j)-I$-generalized $b$-open(in short, $(i, j)-I g b$-open) set if $X \backslash A$ is $(i, j)-I g b$-closed, for $i, j=1,2$ and $i \neq j$.

Theorem 3.5. Let $\left(X, \tau_{1}, \tau_{2}, I\right)$ be an ideal bitopological space. A subset $A$ of $X$ is $(i, j)-I g b$-open in $X$ if and only if $B \backslash P \subset(j, i)-\operatorname{bint}(A)$ for some $P \in I$, whenever $B \subset A$ and $B$ is $\tau_{i}$-closed.

Proof. Let $B \subset A$ and $B$ be $\tau_{i}$-closed. Clearly $X \backslash A \subset X \backslash B$. Since $A$ is $(i, j)-I g b$-open, therefore we have $X \backslash A$ is $(i, j)-I g b$-closed. By definition $(j, i)-b c l(X \backslash A) \backslash(X \backslash B) \in I$. This implies $(j, i)-b c l(X \backslash A) \subset(X \backslash B) \cup P$ for some $P \in I$. This gives that $X \backslash((X \backslash B) \cup P) \subset X \backslash(j, i)-b c l(X \backslash A)$. Thus $B \backslash P \subset X \backslash(X \backslash(j, i)-\operatorname{bint}(A))$ and hence $B \backslash P \subset(j, i)-\operatorname{bint}(A)$.

Conversely suppose that $B \subset A$ and $B$ is $\tau_{i}$-closed. By hypothesis we have $B \backslash P \subset(j, i)-\operatorname{bint}(A)$ where $P \in I$. This implies $B \backslash P \subset$ $X \backslash(j, i)-b c l(X \backslash A)$. Thus $X \backslash(X \backslash(j, i)-b c l(X \backslash A)) \subset X \backslash(B \backslash P)$ and consequently we have $(j, i)-b c l(X \backslash A) \subset(X \backslash B) \cup P$. Hence $(j, i)-b c l(X \backslash A) \backslash(X \backslash B) \in I$ for some $P \in I$. This shows that $X \backslash A$ is $(i, j)-I g b$-closed and so $A$ is $(i, j)-I g b$-open.

Theorem 3.6. Let $\left(X, \tau_{1}, \tau_{2}, I\right)$ be an ideal bitopological space. If $A$ is $(i, j)-I g b$-open in $X$ and $(j, i)$-bint $(A) \subset B \subset A$, then $B$ is $(i, j)-I g b$-open in $X$.

Proof. Assume that $A$ be $(i, j)-I g b$-open. Then $X \backslash A$ is $(i, j)-I g b$-closed. Since $(j, i)-\operatorname{bint}(A) \subset B \subset A$, we have $X \backslash A \subset X \backslash B \subset X \backslash(j, i)$-bint $(A)=$ $(j, i)-b c l(X \backslash A)$. Then by Theorem 3.2 , we have $X \backslash B$ is $(i, j)-I g b$-closed and hence $B$ is $(i, j)-I g b$-open.

Theorem 3.7. The intersection of two $(i, j)-I g b$-open sets in an ideal bitopological space $\left(X, \tau_{1}, \tau_{2}, I\right)$ is also $(i, j)-I g b$-open.

Proof. Suppose $A$ and $B$ be two $(i, j)-I g b$-open sets in $X$. Then $X \backslash A$ and $X \backslash B$ are $(i, j)-I g b$-closed. Now we have $X \backslash(A \cap B)=(X \backslash A) \cup(X \backslash B)$ 
is $(i, j)-I g b$-closed, by Theorem 3.3. Hence $A \cap B$ is $(i, j)$ - $I g b$-open.

Theorem 3.8. Let $\left(X, \tau_{1}, \tau_{2}, I\right)$ be an ideal bitopological space. If $A$ and $B$ are two $(i, j)-I g b$-open sets in $X$ such that $(j, i)-b c l(A) \cap B=\emptyset$ and $A \cap(j, i)-b c l(B)=\emptyset$, then $A \cup B$ is $(i, j)-I g b$-open.

Proof. Let $A$ and $B$ be two $(i, j)-I g b$-open sets in $X$ such that $(j, i)-$ $\operatorname{bcl}(A) \cap B=\emptyset$ and $A \cap(j, i)-b c l(B)=\emptyset$. Suppose $V$ is $\tau_{i}$-closed and $V \subset A \cup B$. Clearly $V \subset A$ and $V \subset B$. Then $V \cap(j, i)-b c l(A) \subset A \cap(j, i)-$ $b c l(A)=A$ and $V \cap(j, i)-b c l(B) \subset B \cap(j, i)-b c l(B)=B$. By hypothesis we have $(V \cap(j, i)-b c l(A)) \backslash P \subset(j, i)-b i n t(A)$ and $(V \cap(j, i)-b c l(B)) \backslash Q \subset$ $(j, i)-\operatorname{bint}(B)$ for some $P, Q \in I$. This implies $(V \cap(j, i)-b c l(A)) \backslash(j, i)-$ $\operatorname{bint}(A) \in I$ and $(V \cap(j, i)-b c l(B)) \backslash(j, i)-\operatorname{bint}(B) \in I$. Then $((V \cap(j, i)$ $b c l(A)) \backslash(j, i)-\operatorname{bint}(A)) \cup((V \cap(j, i)-b c l(B)) \backslash(j, i)-\operatorname{bint}(B)) \in I$. Which implies $(V \cap((j, i)-b c l(A) \cup(j, i)-b c l(B))) \backslash((j, i)-\operatorname{bint}(A) \cup(j, i)-$ $\operatorname{bint}(B)) \in I$. Thus $(V \cap(j, i)-\operatorname{bcl}(A \cup B)) \backslash((j, i)-\operatorname{bint}(A) \cup(j, i)-$ $\operatorname{bint}(B)) \in I$. Further, $V=V \cap(A \cap B) \subset V \cap(j, i)-\operatorname{bcl}(A \cup B)$, we have $V \backslash(j, i)-\operatorname{bint}(A \cup B) \subset(V \cap(j, i)-b c l(A \cup B)) \backslash(j, i)-\operatorname{bint}(A \cup B) \subset$ $(V \cap(j, i)-\operatorname{bcl}(A \cup B)) \backslash((j, i)-\operatorname{bint}(A) \cup(j, i)-\operatorname{bint}(B)) \in I$. This shows that $V \backslash R \subset(j, i)-\operatorname{bint}(A \cup B)$ for some $R \in I$. Hence $A \cup B$ is $(i, j)-I g b$-open.

Theorem 3.9. Let $\left(X, \tau_{1}, \tau_{2}, I\right)$ be an ideal bitopological space. If $A$ is $(i, j)-I g b$-open set relative to $B$ such that $A \subset B \subset X$ and $B$ is $(i, j)-I g b$ open relative to $X$, then $A$ is $(i, j)$ - Igb-open relative to $X$.

Proof. Let $U \subset A$ and $U$ be $\tau_{i}$-closed. Suppose $A$ is $(i, j)-I g b$-open relative to $B$. Then we have $U \backslash P \subset(j, i)-\operatorname{bint}_{B}(A)$ for some $P \in I_{B}$, where $I_{B}$ denotes the ideal of the set $B$. Which implies that there exists a $(j, i)-b$-open set $V_{1}$ such that $U \backslash P \subset V_{1} \cap B \subset A$. Let $U \subset B$ and $U$ is $\tau_{i}$-closed. Suppose $B$ is $(i, j)-I g b$-open relative to $X$. Then we have $U \backslash Q \subset(j, i)-\operatorname{bint}(B)$ for some $Q \in I$. Which implies that there exists a $(j, i)-b$-open set $V_{2}$ such that $U \backslash Q \subset V_{2} \subset B$. Further $\left.U \backslash(P \cup Q)=(U \backslash P) \cap(U \backslash Q) \subset\left(V_{1} \cap B\right) \cap V_{2}\right) \subset\left(V_{1} \cap B\right) \cap B=V_{1} \cap B \subset A$. This shows that $U \backslash(P \cup Q) \subset(j, i)$-bint $(A)$ for some $P \cup Q \in I$. Hence $A$ is $(i, j)-I g b$-open relative to $X$. 


\section{References}

[1] A. A. Abo Khadra and A. A. Nasef, On extension of certain concepts from a topological space to a bitopological space, Proc. Math. Phys. Soc. Egypt 79, pp. 91-102, (2003).

[2] T. Al-Hawary and A. Al-Omari, $b$-open and $b$-continuity in Bitopological Spaces, Al-Manarah, 13 (3), pp. 89-101, (2007).

[3] D. Andrijevic, On b-open sets, Mat. Vesnik,48, pp. 59-64, (1996).

[4] T. Fukutake, On generalized closed sets in bitopological spaces, Bull. Fukuoka Univ. Ed. Part III, 35, pp. 19-28, (1985).

[5] D. Jankovic and T. R. Hamlett, New topologies from old via ideals, Amer. Math. Monthly, 97, pp. 295-310, (1985).

[6] J. C. Kelly, Bitopological spaces, Proc. London Math. Soc., 3 (13), pp. 71-89, (1963).

[7] K. Kuratowski, Topology, Academic Press, New York, (1966).

[8] M. S. Sarsak and N. Rajesh, Special Functions on Bitopological Spaces, Internat. Math. Forum, 4 (36), pp. 1775-1782, (2009).

[9] B. C. Tripathy and B. Hazarika : I-convergent sequence spaces associated with multiplier sequence spaces; Mathematical Inequalities and Applications; 11 (3), pp. 543-548, (2008).

[10] B. C. Tripathy and S. Mahanta : On $I$-acceleration convergence of sequences; Journal of the Franklin Institute, 347, pp. 591-598, (2010). 
[11] B. C. Tripathy and G. C. Ray, On Mixed fuzzy topological spaces and countability, Soft Computing, 16(10), pp. 1691-1695, (2012).

[12] B. C. Tripathy and D. J. Sarma, On b-locally open sets in Bitopological spaces, Kyungpook Math. J., 51(4), pp. 429-433, (2011).

[13] B. C. Tripathy and D. J. Sarma, On pairwise b-locally open and pairwise $b$-locally closed functions in bitopological spaces, Tamkang Jour. Math., 43 (4), pp. 533-539, (2012).

[14] B. C. Tripathy and D. J. Sarma, On weakly b-continuous functions in bitopological spaces, Acta Sci. Technol., 35 (3), pp. 521-525, (2013).

[15] B. C. Tripathy and D. J. Sarma, Generalized b-closed sets in bitopological spaces, (communicated).

[16] B. C. Tripathy, M. Sen and S. Nath : I-convergence in probabilistic n-normed space; Soft Comput., 16, 1021-1027, DOI 10.1007/s00500011-0799-8, (2012).

[17] R. Vaidyanathaswamy, The localization theory in set topology, Proc. Indian Acad. Sci., 20, pp.51-61, (1945).

\section{Binod Chandra Tripathy}

Mathematical Sciences Division

Institute of Advanced Study in Science and Technology

Paschim Boragaon; Garchuk

GUWAHATI - 781035; ASSAM

India

e-mail: tripathybc@yahoo.com tripathybc@rediffmail.com

and 
Diganta Jyoti Sarma

Department of Mathematics

Central Institute of Technology

Kokrajhar-783370

Assam,

India

e-mail: djs_math@rediffmail.com 